\title{
Constructibility of Signal-Crossing Solutions in von Neumann 29-State Cellular Automata
}

\author{
William R. Buckley ${ }^{1}$ and Amar Mukherjee ${ }^{2}$ \\ ${ }^{1}$ California Evolution Institute, San Francisco, CA. 94134 \\ wrb@calevinst.org \\ ${ }^{2}$ Professor of Computer Science, School of Computer Science, \\ University of Central Florida, Orlando, FL. 32816 \\ amarecs.ucf.edu
}

\begin{abstract}
In von Neumann 29-state cellular automata, the crossing of signals is an important problem, with three solutions reported in the literature. These solutions greatly impact automaton design, especially self-replicators. This paper examines these solutions, with emphasis upon their constructibility. We show that two of these solutions are difficult to construct, and offer an improved design technique. We also argue that solutions to the signal-crossing problem have implications for machine models of biological development, especially with regard to the cell cycle.
\end{abstract}

\section{Von Neumann 29-State Cellular Automata Signal-Crossing}

John von Neumann developed cellular automata theory, yielding an environment in which to demonstrate his thesis that machines may be designed having the property of self-replication [1]. Von Neumann cellular automata are characterized by a two-dimensional, rectilinear lattice network of finite state automata (the cells), each identical in form, function, and association, as specified by a set of states, a set of rules for the transition of cells between states (the state transition function), and a grouping function that places each cell at the center of a neighborhood of adjacent cells (specifying the set of cells operated upon by the state transition function in the computation of state transitions). All cells transition their state synchronously.

States are grouped into five categories; a ground state, the transition states, the confluent states (C), the ordinary transmission states $(\rightarrow)$, and the special transmission states $(\Uparrow)$. The last three categories have an activity property, while the last two categories have the property of direction. Activity corresponds to carried data, it being transmitted between states at the rate of one bit per application of the state transition function. Confluent states have the additional property of a one-cycle delay, and so hold two bits of data. The direction property indicates the flow of data between states. Ordinary and special transmission states have an antagonistic relationship, with mutually directed active cells of each causing the annihilation of the other, to yield the ground state. Active special transmission states also yield confluent state annihilation. Confluent states accept data from ordinary transmission states, perform a logical AND on

${ }^{1}$ AKA - Amar Mukhopadhyay

V.S. Sunderam et al. (Eds.): ICCS 2005, LNCS 3515, pp. 395 -403, 2005.

(C) Springer-Verlag Berlin Heidelberg 2005 
the inputs, and transmit data to both ordinary and special transmission states. Ordinary and special transmission states logically OR inputs. An ordinary transmission state accepts input only from like states, and from adjacent confluent states. Special transmission states accept input likewise. Confluent states pass data to any adjacent transmission state not pointed at the confluent state. Data are not transmitted to transmission states against the direction of those transmission states. For instance, two ordinary transmission states pointing at each other do not exchange data. Instead, the data is simply lost. Data held by a confluent state is lost if there is no adjacent transmission state not pointing at the confluent state.

Patterns of cells are called configurations, with those that implement specific functionality being called organs. Configurations can be compared in terms of their constructibility. Constructibility is both an absolute measure, and a relative measure. Some configurations are not constructible, while other configurations are constructible. In absolute terms, constructibility is the property that a configuration can be obtained through the act of another configuration. In relative terms, constructibility is an inverse measure of effort. In von Neumann 29-state cellular automata, the organ that facilitates configuration construction is known as the construction arm.

\section{The Signal-Crossing Problem and Available Solutions}

A problem arises within any two-dimensional system respecting the mechanisms of translocation - the crossing problem. The familiar example is roadway transportation, the solutions being stop-and-go intersections, bridges, and traffic circles. In cellular automata, we have the signal-crossing problem. This owes to the fixed-position nature of the component finite state automata, where the translocation is of data (in the form of signals). In such cases, translocation is called communication.

Signals are an ordered sequence of data (bits), whether of fixed or arbitrary length, that are communicated between organs. The literature reports three solutions to the signal-crossing problem within von Neumann 29-state cellular automata. These signal-crossing organs are the Coded Channel (CC), the Mukhopadhyay Crossing Organ (MCO) [2], and the Real-Time Crossing Organ (RTCO). We are here concerned with the properties of these signal-crossing organs, particularly the latter two.

The MCO and RTCO are general signal-crossing solutions, able to serve the crossing needs of any two signals, regardless of length. The CC is a more constrained signal-crossing solution, capable of serving only signals of varying fixed length, though extendable to service an arbitrarily large number of signals. While the MCO and RTCO are indiscriminate in the signals they service, the $\mathrm{CC}$ discriminates between signals, via selective acceptance. The function of the $\mathrm{CC}$ is programmable, while neither the MCO nor the RTCO is programmable. We now consider signal-crossing organ architecture [3].

The CC has two-layers, with an internal channel (or signal path) positioned between inputs and outputs. The internal channel is of finite length, and is non-cyclic. The first CC layer accepts signal input and translates it into a code carried by the internal 


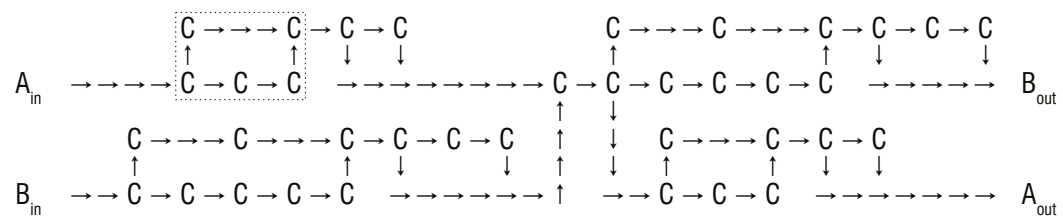

Fig. 1. The minimal $C C$ is a configuration that crosses two signals, $<11>$ and $<101>$. Input $A_{\text {in }}$ is accepted by a decoder/pulser pair, the result being then injected into the internal channel, where an identical decoder/pulser pair again accepts the signal. A single ordinary transmission state separates the decoder from the pulser, each organ being constructed of confluent and ordinary transmission states. The decoder of input $A_{\text {in }}$, outlined in this figure with dashed lines, is an organ of dimension five cells by three cells

channel. The second layer of the $\mathrm{CC}$ translates this code into signal output. The $\mathrm{CC}$ may accept any input signal a multiple number of times, and may generate the corresponding output signal any number of times. Linearity of the internal channel requires input acceptance prior to output generation. Each input may accept more than one signal, while each output generates only one signal. If corruption of channel code occurs, unwanted output signal generation may result. Thus, signal inputs ought occur with sufficient relative delay. The $\mathrm{CC}$ is best applied where it is known that signals are incident only upon the complete servicing of any previously accepted signal.

In the simplest implementation, shown in figure 1, the CC expresses a bijection of two inputs to two outputs. It is especially easy to see in this case that signal input can be both crossed and duplicated on output. If the input signals $A$ and $B$ are accepted in that order, with input acceptors coming before output generators, and the order of outputs is $B$ then $A$, we have that the signals are crossed and duplicated. For signals $<11>$ and $<101>$, the CC covers approximately 230 cells. CC size is proportional to the number and length of inputs and outputs.

The RTCO is a square organ, comprising 64 cells, as shown in figure 2 . It has two inputs and two outputs, arranged in orthogonal input/output pairs. Signals are duplicated at input, routed along a pair of internal paths, and joined into a single signal at output. There are four different signal paths internal to the RTCO, all of identical length.

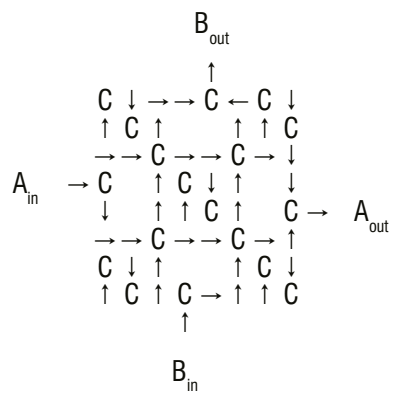

Fig. 2. The RTCO, shown without clock signals, with inputs and outputs indicated 
The RTCO has five clocks, each of identical structure and emitting a period six signal $<101010>$, which drive inputs to outputs and operate in-phase with one-another. Four of these clocks are positioned at the four corners of the RTCO, with the fifth clock located directly in the middle of the RTCO. The four internal signal paths of the RTCO completely surround the central clock. Every other bit of signal input is transmitted along one internal path of the pair while the alternating bits are transmitted along the other path of the pair. Signal transmission through the RTCO is facilitated by the alternating signals generated by the component clocks, which trigger confluent states along the internal paths. These confluent states act as gates to control signal propagation. There are four such gates, each intersecting two internal paths.

Like the RTCO, the MCO has two inputs and two outputs, and the functional elements of the internal paths are similarly co-linear. Unlike the RTCO, the inputs and outputs of the MCO are parallel. The MCO is highly compartmentalised, with a greater variety of functional parts, and exhibits several levels of structure. At the macro-level, the MCO is composed of three units that implement the logical XOR operator, one upstream, and two downstream. The two signals to be crossed are routed through the upstream XOR, the output of which is then routed through the downstream XOR units, each taking as the other input the alternate of the two signals to be crossed. The outputs of these two downstream XOR units will be the two input signals to the MCO, now crossed. A single $X O R$ is shown in figure 3.

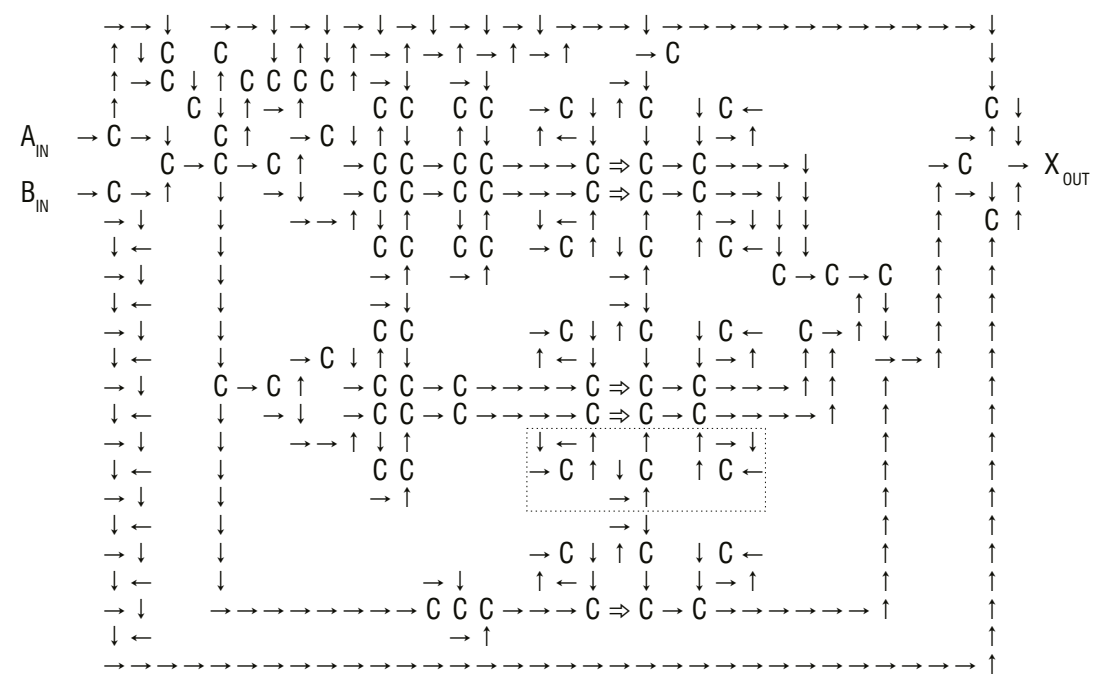

Fig. 3. A single XOR unit of the MCO, shown without clock signals. The five internal paths are clearly visible, as indicated by presence of special transmission states. The clocks of the selector, inverter, collector combination of one path are outlined with a dashed box. The confluent state to the left of the special transmission state is the operational part of the selector, while the confluent state to the right is the operational part of the inverter. The confluent state still further to the right is the operational part of the collector. This organ cannot be autoinitialised 
At the meso-level, each XOR of the MCO is composed of three operational parts, two logical AND operators and a logical negation (NOT) operator, and three internal paths. Each of the two signal inputs to the XOR is first duplicated. One of the duplications of each signal is then routed via an internal path around the outside of the $\mathrm{XOR}$, to its output. The other duplications of the two signals are first combined via a logical AND operator (the two inputs of a confluent state), with the output of the AND then input to the component NOT operator. At output, the result of the NOT is then severally combined with the two duplicated signals to produce the output of the XOR. For the upstream XOR unit, the output is two first-stage (or partially) crossed signals.

At the micro-level, each NOT operator is composed of clocks, three types of gate, and internal paths. There are a total of fifteen clocks per NOT operator, five internal paths, and five of each kind of gate, with each gate being driven by one of the component clocks. Along each internal path, a sequence of the three kinds of gates, together with their driving clocks, is positioned. Each group of three gates implement one NOT operator. The five NOT operators function out-of-phase with each other, staggered to service every fifth bit of signal input. Hence, the clocks emit period five signals. The three gates, in order, select the bit to be inverted (the selector), invert the selected bit (the inverter), and collect the inverted bit (the collector) for transmission to output. The clocks of the selector and collector emit the signal $\langle 10000\rangle$, while the inverter emits the signal $<11111>$. The clock of the collector is out-of-phase with the clock of the selector, following by one state transition.

Given the descriptions above, we can compare the constructibility of the CC, $\mathrm{MCO}$, and RTCO. The single internal path, the lack of interaction between component parts, and the lack of clock organs gives the $\mathrm{CC}$ the highest constructibility. Indeed, the presence of clock organs is the most important determinant of the unconstructibility of signal-crossing organs. The reason is simple: clocks are active organs. None of the component organs of the $\mathrm{CC}$ is active, while the MCO and RTCO each have several active component organs. We now look at the effect of active organs upon constructibility.

\section{Configuration Construction}

Constructibility is strongly impacted by configuration activity and the phasing of component clocks. All passive configurations are constructible. Unconstructible configurations express a signal, as suggested in figure 4. Yet, not all configurations that express a signal are unconstructible. In practice, the unconstructibility of a configuration has as much to do with the construction arm as with the configuration.

Configuration construction involves the sequential construction of individual cells, requiring a signal of between four and five bits, depending upon the desired end state of the constructed cell. For instance, confluent cell construction requires signal $<1111>$ while construction of a right-pointing ordinary transmission cell requires signal $<10000>$. At least four ticks of the system clock are required for the construction of a single cell. Another time cost to construction is the motion of the construction arm. The 


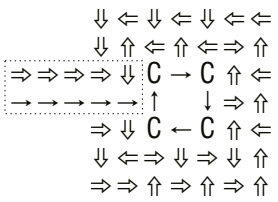

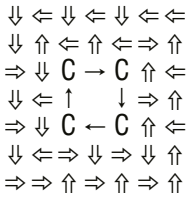

Fig. 4. An unconstructible configuration, shown without signaling, in both expected final form (right), and during construction (left), where the clock signal is the twelve-bits $<111111111111$. Construction fails upon attempt to initialise the clock via the ordinary transmission path of the construction arm: interference from the clock alters the signaling of the construction arm. The construction arm is outlined with a dashed box

process of construction begins with the extension of the construction arm, followed by a sequence of pairings of cell constructions and construction arm retractions, one pair per cell constructed. Extension of the construction arm requires the separate construction of four cells, and the annihilation of two cells (a minimum unit cost of 18 system clock ticks), while retraction requires the separate construction of seven cells, and annihilation of nine cells (a minimum unit cost of 37 system clock ticks). Construction proper occurs during construction arm retraction, so construction of a single cell requires at least 41 system clock ticks.

The proximity of an active clock may impede cell construction. This occurs in two ways. Either the clock interferes with the signaling of the construction arm, or the clock provides alternative signaling to the constructed cell. In general, the signal emitted by a clock is not the same as the signal used to construct a cell. Similarly, the emitted signal will not likely correspond to the signal needed for construction arm extension and retraction. The far more likely condition is that the emitted signal will corrupt the signaling of the construction arm, or alter the state of a constructed cell. Interference with the construction arm is the reason that the configuration shown in figure 4 is unconstructible.

Even where an active configuration is constructible, the presence of multiple clocks presents a special difficulty. The construction arm can perform only one operation at a time, such as passing a signal to a clock. Consider the case in which two clocks are initialised, with one clock already circulating its signal. In order for the second clock to remain in proper relative phasing, the signal to the construction arm must be accurately timed. This requires knowledge of the phasing of the already operating clock, the length of the construction arm, and perhaps other quantities.

One means to construct a configuration having active organs is the use of a dedicated pulser. This allows the a priori computation of proper signaling for the construction arm, and so ensures proper clock phasing. For the RTCO, the configuration size of this pulser is some $10^{5}$ cells, three orders of magnitude larger than the configuration size of the RTCO. To a good approximation, such a pulser would produce a signal of some $10^{4}$ bits. $^{2}$ The size of this dedicated pulser is some ten percent of the size of a complete self-replicator, sans the external store [4]. The phasing of

\footnotetext{
${ }^{2}$ Though we have not sufficient room in this paper to demonstrate the result, it has been computed.
} 
multiple clocks is not at all a trivial problem, the MCO and RTCO being good examples. Through redesign, both the RTCO and MCO can be made more constructible, which we now demonstrate for the MCO.

\section{Autoinitialisation}

Mukhopadhyay anticipated the difficulty of clock phasing in signal-crossing configuration construction, suggesting that means might exist to set the timing of clocks as a consequence of configuration function. The technique involves the sampling of signal input, and using the sample to trigger component clocks. We call this method autoinitialisation, and recognise the inherent implications of the technique for machine models of biological development, with particular respect to control of gene expression.

Configurations capable of autoinitialisation include subconfigurations (or AI organs) dedicated to the process. These AI organs sample signal inputs to the configuration, and generate control and clock signals. For many configurations, autoinitialisation is a one-time operation, occuring only for the first input signal. We now consider the case of an MCO configuration that supports autoinitialisation, and note one AI organ per internal path. Figure 5 shows the design of an MCO that includes

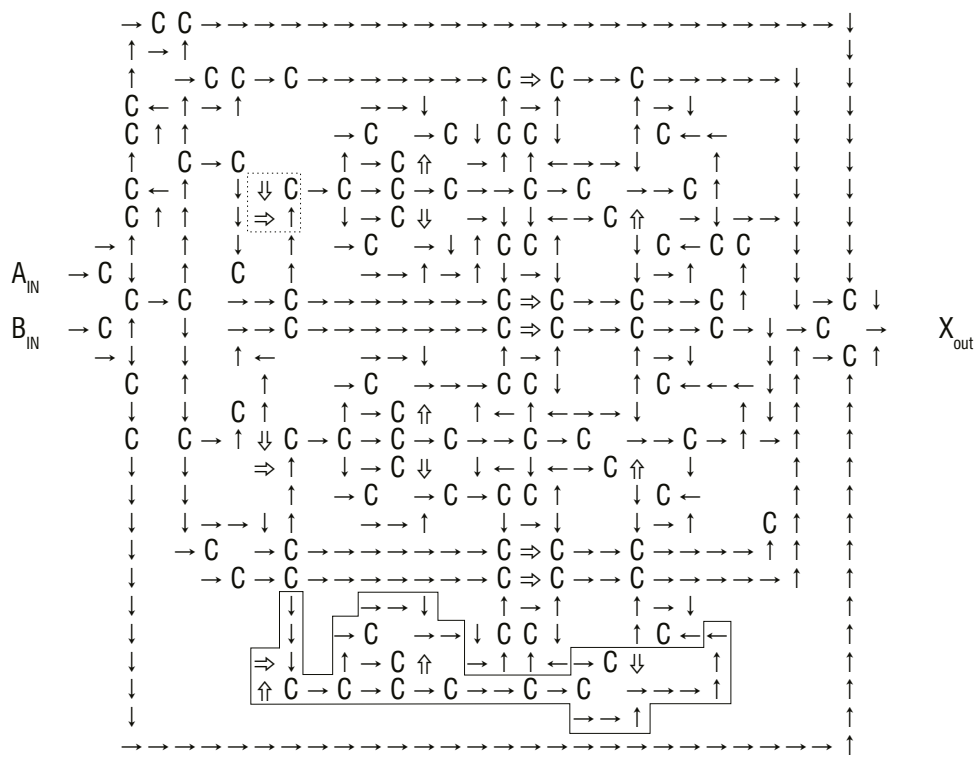

Fig. 5. An XOR unit of the MCO that supports autoinitialisation. Portals are indicated by two adjacent special transmission states, with one outlined with a dashed box. The autoinitialisation organ of one signal path is outlined with a solid line, at the bottom of the figure. The configuration shown is autoinitialised with the signal $<11111>$, applied synchronously at both inputs 
AI organs. Each AI organ obtains signal input from the adjacent internal path of the MCO through a portal, generates properly phased signals for the component clocks of the corresponding selector, inverter, and collector gates, and generates a signal that closes the portal. Each portal consists of a confluent state inserted into the corresponding internal path. This confluent state duplicates the signal carried by the internal path, transmitting it both along the internal path, and to the AI organ, via adjacent ordinary transmission states that are pointed away from and perpendicular to the internal path. The portal is closed by changing this perpendicular ordinary transmission state into a confluent state. Closing the portal ensures that subsequent inputs to the configuration do not corrupt the signals of the clocks.

In addition to the increase in configuration size implied by the inclusion of AI organs, there is a cost to the time performance of the configuration. This cost comes in one of two forms. There is either a latency time cost or a propagation time cost associated with configuration function. In the case where the autoinitialisation is triggered by a dedicated signal, a design based upon latency is preferred, resulting in a one-time delay to the use of configuration function. If a dedicated signal is not available, then a design based upon propagation is the appropriate choice, with the delay borne for every use of the configuration. We term post construction changes to a configuration a reconfiguration. The MCO reconfigurations described are fairly simple to implement. Reconfigurations can be much more complex operations, involving dynamic change to a configuration, and they may be linked hierarchically.

One expects many layers of autoinitialisation to provide examples of machine-modeled biological development. For instance, the sequence of events in the cell cycle of bacteria consists of three basic phases: regeneration, regulation, and replication. The mechanisms of autoinitialisation suggest cellular automata replication can be extended into the processes of regeneration and regulation. Thus, with cascades of autoinitialisation giving rise to change in the function of a configuration, we can see opportunity for modeling replication in terms of regulation, instead of as a stand-alone (holistic) process. Further, if some operations in the cascade result in the expression of organs not present at replication, then regeneration becomes apparent. It is not so hard to envision a configuration that functions through such a sequence, thereby expressing a crude model of the cell development process.

\section{Conclusions}

As we have shown, solutions to the signal crossing problem in von Neumann cellular automata are hard to construct. We have developed the technique of autoinitialisation, and have demonstrated its application, yielding great improvement in configuration constructibility. For instance, the technique is easily applied to the task of postconstruction initiation of clocks. Autoinitialisation can be extended to facilitate the run-time alteration of configurations. Systems of hierarchical autoinitialisation have strong implications for the modeling of the cell cycle. We expect that extension of the ideas presented here will yield computational models of biological developmental processes. 


\section{References}

1. von Neumann, J.: Theory of Self-Reproducing Automata. University of Illinois Press, Urbana and London (1966)

2. Mukhopadhyay, A. Representation of Events in the von Neumann Cellular Model. J. of the ACM, Vol. 15, No. 4, October 1968, pp. 693-705

3. Burks, A. W. (ed.): Essays on Cellular Automata. University of Illinois Press, Urbana and London (1970)

4. Mange, D., Stauffer, A., Peparolo, L., Tempesti, G.: A Macroscopic View of SelfReplication. Proc. of the IEEE, Vol. 92, No. 12, December 2004, pp. 1929-1945 